\title{
ON 6-CANONICAL MAP OF IRREGULAR THREEFOLDS OF GENERAL TYPE
}

\author{
Jungkai Chen, Meng Chen and Zhi Jiang
}

\begin{abstract}
We prove that, for any non-singular projective irregular threefold of general type, the 6-canonical map is birational onto its image.
\end{abstract}

\section{Introduction}

Given a non-singular projective variety $V$ of general type, by definition, the pluricanonical map $\varphi_{m}$ is birational for all sufficiently large integer $m$. It is natural and interesting to find an effective bound for $m$. By the result of Hacon-McKernan [8], Takayama [11] and Tsuji (cf. [12]), one knows that there exists a positive integer $r_{n}$ depending only on $n=\operatorname{dim}(V)$ such that $\varphi_{m}$ is birational for all $m \geq r_{n}$. In the case of threefolds, the previous work of the first two authors (cf. [3,4]) shows that $r_{3} \leq 73$.

In this paper, we study irregular threefolds (i.e. $q(V)>0$ ) of general type. Recent developments on the technique inspired by the Fourier-Mukai transform show that the geometry of irregular threefolds is very similar to that of general fibers of the Albanese map. Noting that the five-canonical map of a general type surface is birational, one may expect that $\varphi_{5}$ is birational too for those threefolds, which admit a fibration over (a subvariety of) an abelian variety. Indeed, given a non-singular projective irregular threefold of general type, it has been proved by Chen and Hacon [6, Theorem 2.8, Proposition 2.9] that $\varphi_{m}$ is birational for all $m \geq 7$ and, moreover, that $\varphi_{5}$ is birational if $\chi\left(\omega_{X}\right)>0$.

The aim of this paper is to prove the following:

Theorem 1.1. Let $V$ be a non-singular projective irregular threefold of general type. Then $\varphi_{6}$ is birational.

\section{Proof of the main theorem}

2.1. Reductions. In order to prove Theorem 1.1, we have the following reduction to special cases:

(1) Let $V$ be a non-singular projective threefold of general type. Take any birational projective model $W$ of $V$ so that $W$ has at worst canonical singularities. Then $V$ and $W$ share the same birational invariants and $\Phi_{m K_{W}} \approx \Phi_{m K_{V}}$. Therefore, it is sufficient to prove the statement of Theorem 1.1 just replacing $V$ with any suitable birational model $W$. 
(2) By Chen and Hacon [6, Proposition 2.9], one only needs to consider the following situation (since, otherwise, $|6 K|$ gives a birational map):

(দ) The Albanese map of $V$ induces the fibration $a_{V}: V \longrightarrow C$ onto an elliptic curve $C$, of which the general fiber is a $(1,2)$ surface $S$, i.e., $\left(K_{S_{0}}^{2}, p_{g}(S)\right)=(1,2)$, where $S_{0}$ is assumed to be the minimal model of $S$.

(3) Also due to Chen and Hacon [6, Theorem 1.1], we may assume that $\chi\left(\mathcal{O}_{V}\right) \geq 0$ (since, otherwise, $|5 K|$ gives a birational map).

(4) By running the minimal model program, one obtains a relative minimal model $X \rightarrow C$ of $a_{V}$ where $X$ has $\mathbb{Q}$-factorial terminal singularities. Then $K_{X / C}$ is nef (see, for instance, Ohno [9, Theorem 1.4]), which means that $X$ is minimal since $K_{C}$ is trivial. In the proof of Theorem 1.1, we may and do replace $V$ by a minimal model $X$ (i.e., $K_{X}$ nef), which has at worst $\mathbb{Q}$-factorial terminal singularities.

Corollary 2.1. Suppose $V$ (or $X$ ) satisfies 2.1(2) and 2.1(3). Then $q(X)=1$, $p_{g}(X)=h^{2}\left(\mathcal{O}_{X}\right) \leq 2$ and thus $\chi\left(\mathcal{O}_{X}\right)=0$.

Proof. Clearly one has $q(V)=1$. Since $q(S)=0$, we see $h^{2}\left(\mathcal{O}_{V}\right)=h^{1}\left(a_{*} \omega_{V}\right)$. So one has $\chi\left(\mathcal{O}_{V}\right)=h^{2}\left(\mathcal{O}_{V}\right)-p_{g}(V)=h^{1}\left(a_{*} \omega_{V}\right)-h^{0}\left(a_{*} \omega_{V}\right)=-\operatorname{deg}\left(a_{*} \omega_{V / C}\right) \leq 0$ by the semi-positivity theorem of Fujita [7]. Thus $\chi\left(\mathcal{O}_{V}\right)=0$ and $p_{g}(V)=h^{2}\left(\mathcal{O}_{V}\right)$. Also by the semi-positivity of $a_{*} \omega_{V}=a_{*} \omega_{V / C}, p_{g}(V)=h^{2}\left(\mathcal{O}_{V}\right)=h^{1}\left(a_{*} \omega_{V}\right) \leq \operatorname{rk}\left(a_{*} \omega_{V}\right)=2$. By Reid's R-R formula in [10], one can see $P_{2}(V)>0$ and $P_{m+1}(V)>P_{m}(V)$ for all $m \geq 2$.

2.2. Definitions and lemmas. Before proving the main result, we would like to recall some notion and results in Chen and Hacon [6].

Definition 2.2. For any vector bundle $E$ on an elliptic curve, we write $E=\oplus E_{i}$, where each $E_{i}$ is indecomposable. We define $\nu(E):=\min \left\{\mu\left(E_{i}\right)\right\}$, where $\mu\left(E_{i}\right)=$ $\frac{\operatorname{deg}\left(E_{i}\right)}{\operatorname{rk}\left(E_{i}\right)}$ is the slope of $E_{i}$.

Definition 2.3. A coherent sheaf $\mathcal{F}$ on an abelian variety $A$ is said to be $I T^{0}$ if $H^{i}(A, \mathcal{F} \otimes P)=0$, for all $i>0$ and all $P \in \operatorname{Pic}^{0}(A)$.

Remark 2.4 (see e.g. [5, Lemma 2.1]). Given a non-zero $I T^{0}$ sheaf $\mathcal{F}$ on an elliptic curve, it is clear that $h^{0}(A, \mathcal{F}) \geq 1$ and hence $\nu(\mathcal{F}) \geq \frac{1}{\operatorname{rk}(\mathcal{F})}$.

Lemma 2.5 ( [6, Lemma 4.8]). Let $E_{1}, E_{2}$ be vector bundles on an elliptic curve.

(1) If $E_{1}, E_{2}$ are indecomposable and $\operatorname{Hom}\left(E_{1}, E_{2}\right) \neq 0$, then $\mu\left(E_{2}\right) \geq \mu\left(E_{1}\right)$.

(2) If there exists a surjective map $E_{1} \rightarrow E_{2}$, then $\nu\left(E_{2}\right) \geq \nu\left(E_{1}\right)$.

Lemma 2.6 ( $\left[\mathbf{6}\right.$, Lemma 4.10]). Let $E$ be an $I T^{0}$ vector bundle on an elliptic curve, which admits a short exact sequence

$$
0 \rightarrow F \rightarrow E \rightarrow Q \rightarrow 0
$$

of coherent sheaves such that $Q$ has generic rank $=0$ (resp. $\leq 1)$. Then $\nu(E) \geq \nu(F)$ (resp. $\nu(E) \geq \min \{1, \nu(F)\})$. 
2.3. Multiplication maps $\boldsymbol{\varphi}_{\boldsymbol{m}, \boldsymbol{n}}$ and $\boldsymbol{\psi}_{\boldsymbol{m}, \boldsymbol{n}}$. Consider the fibration $a: X \rightarrow C$ as in 2.1. Let $F$ be a general fiber of $a$. Let $R_{m}:=H^{0}\left(F, \omega_{F}^{m}\right)$ and $E_{m}:=a_{*} \omega_{X}^{m}$. By Chen and Hacon [6, Lemma 4.1], $E_{m}$ is an $I T^{0}$ vector bundle of rank $P_{m}(F)$ for all $m \geq 2$. We also remark that $\nu\left(E_{m}\right) \geq 0$ by the semi-positivity theorem (see, for instance, Viehweg [13]) and Atiyah's description of vector bundles over elliptic curves (cf. [1]). We consider the multiplication map of pluricanonical systems on the fiber $F$, say

$$
\varphi_{m, n}: R_{m} \otimes R_{n} \rightarrow R_{m+n} .
$$

This naturally induces a map between vector bundles

$$
\psi_{m, n}: E_{m} \otimes E_{n} \rightarrow E_{m+n},
$$

where $m, n>0$. Clearly if cokernel of $\varphi_{m, n}$ has dimension $\leq r$, then cokernel of $\psi_{m, n}$ has rank $\leq r$.

2.4. Proof of Theorem 1.1. First of all, we recall that the linear system $\left|6 K_{V}\right|$ separates two general points on two distinct general fibers of the Albanese map $a_{V}$ (see [6, Theorem $2.8(2)]$ ). Hence, we just need to show that $\left|6 K_{V}\right|$ separates two general points on a general fiber of $a_{V}$ to conclude the proof of Theorem 1.1.

We now take the birational model $a: X \rightarrow C$ of $V$ as in 2.1(1) (4) and denote by $F$ a general fiber of $a$.

Step 1. We consider the relative canonical model

$$
W:=\operatorname{Proj} \bigoplus_{m} a_{*} \omega^{m} \stackrel{g}{\rightarrow} C,
$$

where $\omega:=\mathcal{O}_{X}\left(K_{X}\right)$. Let $G$ be a general fiber of $g$. It is known (cf. Catanese $[2,1.3$ Example]) that the canonical model $G$ of any $(1,2)$ surface is a degree 10 weighted hypersurface, with at worst rational double points, in $\mathbb{P}(1,1,2,5)$. Namely, if $x, y, z, u$ are coordinates of $\mathbb{P}(1,1,2,5)$, then $G$ is given by the homogeneous equation $u^{2}-f_{10}(x, y, z)$ for some homogeneous polynomial $f_{10}(x, y, z)$ of degree 10 in $x, y, z$. Furthermore the bicanonical map $\varphi_{2}$ of $G$ is a double covering onto $\mathbb{P}(1,1,2)$ branched along a reduced divisor $B_{0}=\operatorname{div}\left(f_{10}\right) \subset \mathbb{P}(1,1,2)$ of degree 10 .

Step 2. The relative bicanonical map $h: Y \rightarrow C$ of $g$.

We start by considering $\mathbb{P}\left(a_{*} \omega^{2}\right)=\mathbb{P}\left(E_{2}\right)$, which is a $\mathbb{P}^{3}$-bundle over $C$. Let $W \rightarrow$ $\mathbb{P}\left(E_{2}\right)$ be the induced map. Take $Y$ to be the closure of $W_{\eta} \rightarrow \mathbb{P}\left(E_{2}\right)_{\eta}$, where $\eta$ is the generic point of $C$. We have a fibration $h: Y \rightarrow C$.

Let $H$ be a general fiber of $h: Y \rightarrow C$. Over a general point of $C$, we have morphisms $F \rightarrow G \rightarrow H$ where $F$ is a minimal (1,2) surface, $G$ is the degree 10 hypersurface in $\mathbb{P}(1,1,2,5)$ with $\mathrm{RDPs}$ and $H \cong \mathbb{P}(1,1,2)$. It is straightforward to see that both $X \rightarrow Y$ and $W \rightarrow Y$ are well-defined over general points of $C$. Replacing both $X$ and $W$ with suitable birational models $\hat{X}$ and $\hat{W}$ by a necessary birational modification to those indeterminancies, we have the following commutative diagram:

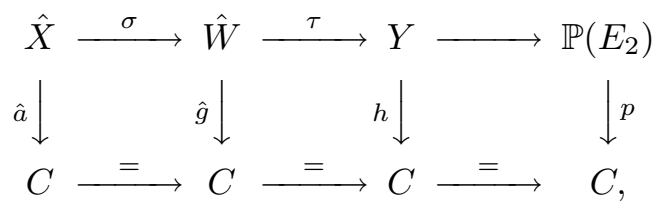


where $\hat{X}$ (resp. $\hat{W}$ ) coincides with $X$ (resp. $W$ ) over a Zariski open subset $U$ of $C$ and $\hat{a}$ (resp. $\hat{g}$ ) factors through $a$ (resp. $g$ ).

Step 3. The decomposition of $E_{m}$ by the double covering construction.

Let $B$ be the branched divisor of $\hat{W} \rightarrow Y$ and let $\mu: \tilde{Y} \rightarrow Y$ be the log resolution of $(Y, B)$. We take $\tilde{X}$ to be the normalization of $\tilde{Y}$ in $K(X)$. One sees that $\tilde{X}$ has at worst canonical singularities by local consideration. We have the double covering $\tilde{\pi}: \tilde{X} \rightarrow \tilde{Y}$ corresponding to the data $(\tilde{B}, \tilde{\mathcal{L}})$. We thus have

$$
\tilde{\pi}_{*} \mathcal{O}_{\tilde{X}}\left(m K_{\tilde{X}}\right)=\mathcal{O}_{\tilde{Y}}\left(m K_{\tilde{Y}}\right) \otimes \tilde{\mathcal{L}}^{m} \oplus \mathcal{O}_{\tilde{Y}}\left(m K_{\tilde{Y}}\right) \otimes \tilde{\mathcal{L}}^{m-1}
$$

for all $m>0$. Now if we take a common birational modification to both $\hat{X}$ and $\tilde{X}$ and take push-forwards in two directions, respectively, we shall get the following decomposition:

$$
E_{m}:=E_{m, 0} \oplus E_{m, 1}
$$

where

$$
\begin{aligned}
E_{m} & :=a_{*} \mathcal{O}_{X}\left(m K_{X}\right) ; \\
E_{m, 0} & :=h_{*} \mu_{*}\left(\mathcal{O}_{\tilde{Y}}\left(m K_{\tilde{Y}}\right) \otimes \tilde{\mathcal{L}}^{m}\right) ; \\
E_{m, 1} & :=h_{*} \mu_{*}\left(\mathcal{O}_{\tilde{Y}}\left(m K_{\tilde{Y}}\right) \otimes \tilde{\mathcal{L}}^{m-1}\right) .
\end{aligned}
$$

Step 4. Calculating $\nu\left(E_{6, i}\right)$.

It is rather easy to check that $\operatorname{rk}\left(E_{m, 0}\right)=h^{0}(H, \mathcal{O}(m))$ and $\operatorname{rk}\left(E_{m, 1}\right)=h^{0}(H$, $\mathcal{O}(m-5))$ for a general fiber $H$ of $h$. Indeed, for $t \in U$,

$$
\begin{gathered}
E_{m, 0} \otimes k(t) \cong H^{0}\left(H_{t}, \mathcal{O}_{H_{t}}\left(m K_{Y}+m L\right)\right) \cong H^{0}(\mathbb{P}(1,1,2), \mathcal{O}(m)) \text { and } \\
E_{m, 1} \otimes k(t) \cong H^{0}\left(H_{t}, \mathcal{O}_{H_{t}}\left(m K_{Y}+(m-1) L\right)\right) \cong H^{0}(\mathbb{P}(1,1,2), \mathcal{O}(m-5)) . \\
E_{m} \otimes k(t) \cong H^{0}\left(F_{t}, \mathcal{O}_{F_{t}}\left(m K_{X}\right)\right) \cong H^{0}\left(G_{t}, \mathcal{O}_{G_{t}}\left(m K_{W}\right)\right) \\
\cong H^{0}(\mathbb{P}(1,1,2), \mathcal{O}(m) \oplus \mathcal{O}(m-5)) .
\end{gathered}
$$

It follows that $\psi_{m, n}$ induces a map

$$
E_{m, 0} \otimes E_{n, 0} \rightarrow E_{m+n, 0} .
$$

Since $E_{m, 0}=E_{m}$ for $m \leq 4$. One sees that

$$
\psi_{4,2}: E_{4} \otimes E_{2} \cong E_{4,0} \otimes E_{2,0} \rightarrow E_{6,0}
$$

is generically surjective. Since $E_{2}$ is a non-zero $I T^{0}$ sheaf, we have $h^{0}\left(E_{2}\right) \geq 1$. Hence, $\nu\left(E_{2}\right) \geq \frac{1}{4}$. Since $\psi_{2,2}$ is generically surjective, we have $\nu\left(E_{4}\right) \geq 2 \nu\left(E_{2}\right) \geq \frac{1}{2}$ by Lemma 2.6. Similarly, $\nu\left(E_{6,0}\right) \geq \frac{3}{4}$. Moreover, $E_{6,1}$ is $I T^{0}$ of rank 2 ; therefore $\nu\left(E_{6,1}\right) \geq \frac{1}{2}$ (cf. Remark 2.4).

Step 5. Birationality of $\varphi_{6}$.

We need the following:

Lemma 2.7. Let $\mathcal{F}$ be a coherent sheaf on $X$ and $\mathcal{E}:=a_{*} \mathcal{F}$ on $C$. Suppose that $\mathcal{E}$ is an $I T^{0}$ vector bundle. Then for any general fiber $X_{t}$, the image of the restriction map $H^{0}(C, \mathcal{E}) \cong H^{0}(X, \mathcal{F}) \stackrel{\text { res }}{\rightarrow} H^{0}\left(X_{t},\left.\mathcal{F}\right|_{X_{t}}\right)$ has dimension $\geq \operatorname{rk}(\mathcal{E}) \cdot \min \{\nu(\mathcal{E}), 1\}$. 
Proof. Take the decomposition of $\mathcal{E}=\oplus \mathcal{E}_{i}$ into indecomposable bundles. For each $i$, there is an induced exact sequence

$$
0 \rightarrow \mathcal{E}_{i} \otimes \mathcal{O}_{C}(-t) \rightarrow \mathcal{E}_{i} \rightarrow \mathcal{E}_{i} \otimes k(t) \rightarrow 0
$$

Let $d_{i}=\operatorname{deg}\left(\mathcal{E}_{i}\right)$ and $r_{i}=\operatorname{rk}\left(\mathcal{E}_{i}\right)$, then $\mathcal{E}_{i} \otimes \mathcal{O}_{C}(-t)$ has rank $r_{i}$ and degree $d_{i}-r_{i}$. If $d_{i}=r_{i}$, then $\mathcal{E}_{i} \otimes \mathcal{O}_{C}(-t)$ is a indecomposable rank $r_{i}$ vector bundle of degree 0. Hence, $\mathcal{E}_{i} \otimes \mathcal{O}_{C}(-t) \cong U_{r_{i}} \otimes P$ for some $P \in \operatorname{Pic}^{0}(C)$ and $U_{r_{i}}$ is a unipotent vector bundle (cf. [1]). Whenever $P=\mathcal{O}$, we pick $t^{\prime} \neq t$ and consider $\mathcal{E}_{i} \otimes \mathcal{O}_{C}\left(-t^{\prime}\right) \cong$ $U_{r_{1}} \otimes \mathcal{O}\left(-t^{\prime}+t\right)$ instead so that it has no global section. Hence, we may and do assume that $H^{0}\left(\mathcal{E}_{i} \otimes \mathcal{O}_{C}(-t)\right)=0$ for general $t \in C$ if $d_{i}=r_{i}$.

It now follows that $h^{0}\left(\mathcal{E}_{i} \otimes \mathcal{O}_{C}(-t)\right)=\max \left\{0, d_{i}-r_{i}\right\}$ for general $t$. Hence, the image of $H^{0}\left(\mathcal{E}_{i}\right) \rightarrow H^{0}\left(\mathcal{E}_{i} \otimes k(t)\right)$ has dimension $d_{i}$ (resp. $\left.r_{i}\right)$ if $d_{i}<r_{i}$ (resp. $d_{i} \geq r_{i}$ ). The statement now follows by simply taking the sum.

Let $V_{m, i}(i=0,1)$ be the image of the following map

$$
\left.H^{0}\left(C, E_{m, i}\right)\right) \hookrightarrow H^{0}\left(C, E_{m}\right) \stackrel{\text { res }}{\rightarrow} H^{0}\left(F_{t},\left.\mathcal{O}(m K)\right|_{F_{t}}\right)
$$

for a general point $t \in C$. Then we have $\operatorname{dim} V_{6,0} \geq 12$ and $\operatorname{dim} V_{6,1} \geq 1$ by Lemma 2.7 . Claim. The subsystem given by the vector space

$$
V_{6,0}+V_{6,1} \subset H^{0}\left(G_{t}, \mathcal{O}(6)\right)
$$

gives a birational map on $G_{t}$ for all general $t \in C$.

We consider the local sections explicitly. Let $x, y, z, u$ be all the 4 coordinates of $\mathbb{P}(1,1,2,5)$ with weights $1,1,2,5$. Then $E_{m, 0} \otimes k(t)$ is generated by sections in $\left\{x^{i} y^{j} z^{k} \mid i+j+2 k=m\right\}$ and $E_{m, 1} \otimes k(t)$ is generated by sections in $\left\{x^{i} y^{j} z^{k} u \mid i+j+\right.$ $2 k=m-5\}$. In a word, either $x u$ or $y u$ extends to global sections in $H^{0}\left(X, 6 K_{X}\right)$. Furthermore, at least 12 linearly independent sections in $E_{m, 0} \otimes k(t)$ can be extended to global sections in $H^{0}\left(X, 6 K_{X}\right)$.

To prove the claim, we put $H=H_{t}$ and let $\Sigma_{0} \subset H^{0}\left(H, \mathcal{O}_{H}(6)\right)$ (resp. $\Sigma_{1} \subset H^{0}$ $\left.\left(H, \mathcal{O}_{H}(6)\right)\right)$ be the subspace spanned by $\left\{x^{6}, \ldots, y^{6}\right\}$ (resp. by $\left.\left\{x^{4} z, x^{3} y z, \ldots, y^{4} z\right\}\right)$. We see that $\operatorname{dim} \Sigma_{0}=7$ and $\operatorname{dim} \Sigma_{1}=5$. By dimensional considerations, one has $\operatorname{dim} V_{6,0} \cap \Sigma_{0} \geq 3$ and $\operatorname{dim} V_{6,0} \cap \Sigma_{1} \geq 1$. Pick linearly independent elements $\sigma_{0,1}, \sigma_{0,2}$, $\sigma_{0,3} \in V_{6,0} \cap \Sigma_{0}$ and $z \sigma_{1} \in V_{6,0} \cap \Sigma_{1}$. We consider the map $\tilde{\varphi}: H \rightarrow \mathbb{P}^{3}$ defined by these 4 sections. Since the map on $H$ defined by $\left(\sigma_{0,1}: \sigma_{0,2}: \sigma_{0,3}\right)$ has image of dimension at least 1 and $z \sigma_{1}$ is algebraically independent of $\sigma_{0,1}, \sigma_{0,2}, \sigma_{0,3}$ in the function field of $H, \tilde{\varphi}$ has image of dimension 2 . We then consider the map $\varphi: H \rightarrow$ $\mathbb{P}^{11}$ given by $V_{6,0}$ with image $H^{\prime}$. Since $\varphi$ factors through $\tilde{\varphi}$ on a non-empty Zariski open subset of $H$, one sees that $H^{\prime}$ is a surface and clearly $\operatorname{deg}\left(H^{\prime}\right) \geq 10$. Since

$$
\operatorname{deg}(\varphi) \cdot \operatorname{deg}\left(H^{\prime}\right) \leq\left(\mathcal{O}_{H}(6) \cdot \mathcal{O}_{H}(6)\right)_{H}=18,
$$

it follows that $\operatorname{deg}(\varphi)=1$, hence $\varphi$ is birational.

Since $G_{t} \cong X_{10} \rightarrow \mathbb{P}(1,1,2) \cong H$ is a $2: 1$ map and $u$ can separate points on general fibers of this double covering. Hence, the sections in $V_{6,1}$ separate points on general fibers of this double covering.

The Claim now follows and hence this completes the proof of Theorem 1.1. 
Example 2.8. Suppose that there exists a minimal irregular threefold $X$ with a fibration $f: X \rightarrow C$ fibered by $(1,2)$ surfaces. Suppose that $K_{X}^{3}=\frac{1}{2}$ and $B(X)=\{3 \times(1,2)\}$. By Reid's $\mathrm{R}-\mathrm{R}$ formula, one has $P_{2}(X)=1, P_{3}(X)=2$, $P_{4}(X)=5, P_{5}(X)=9$ and $P_{6}(X)=16$. We show that $\left|5 K_{X}\right|$ may be non-birational.

Note that $\operatorname{rk}\left(E_{5,0}\right)=12$ and $\operatorname{rk}\left(E_{5,1}\right)=1$. Assume $h^{0}\left(E_{5,0}\right)=8$ and $h^{0}\left(E_{5,1}\right)=1$.

Now $H^{0}\left(F_{t},\left.5 K\right|_{F_{t}}\right)$ is generated by

$$
\left\{x^{5}, \ldots, y^{5}, x^{3} z, x^{2} y z, x y^{2} z, y^{3} z, x z^{2}, y z^{2}, u\right\} .
$$

If $V_{5,0}$ is generated by $\left\{x^{5}, \ldots, y^{5}, x z^{2}, y z^{2}\right\}$ and $V_{5,1}$ is generated by $u$, then these sections cannot distinguish points $\left(x_{0}, y_{0}, z_{0}, u_{0}\right)$ from $\left(x_{0}, y_{0},-z_{0}, u_{0}\right)$. In other words, $\left|V_{5,0}+V_{5,1}\right|$ may only give a $2: 1$ map on $F_{t}$ instead of a birational map.

However, we do not know whether this kind of examples really exists or not.

\section{Acknowledgments}

Jungkai Chen was partially supported by the NCTS/TPE and National Science Council of Taiwan. Meng Chen was supported by the National Natural Science Foundation of China (grant no. 11171068), Doctoral Fund of Ministry of Education of China (grant no. 20110071110003) and partially by the NSFC for Innovative Research Groups (grant no. 11121101).

\section{References}

[1] M. Atiyah, Vector bundles over an elliptic curves, Proc. London Math. Soc. 7 (1957), 414-452.

[2] F. Catanese, Canonical rings and "special" surfaces of general type. Algebraic geometry, Bowdoin, 1985, Brunswick, Maine, 1985, 175-194; (Proc. Symp. Pure Math., 1987) 46, Part 1, Amer. Math. Soc., Providence, RI.

[3] J.A. Chen and M. Chen, Explicit birational geometry of threefolds of general type, I, Ann. Sci. Ecole Norm. Sup. 43 (2010), 365-394.

[4] J.A. Chen and M. Chen, Explicit birational geometry of threefolds of general type, II, J. Diff. Geom. 86 (2010), 237-271.

[5] J.A. Chen and C.D. Hacon, Linear series of irregular varieties, Algebraic geometry in East Asia, Kyoto, 2001, 143-153; World Sci. Publ., River Edge, NJ, 2002.

[6] J.A. Chen and C.D. Hacon, Pluricanonical systems on irregular 3-folds of general type. Math. Z. 255(2) (2007), 343-355.

[7] T. Fujita, On Kaehler fiber spaces over curves. J. Math. Soc. Japan 30(4) (1978), 779-794.

[8] C.D. Hacon and J. McKernan, Boundedness of pluricanonical maps of varieties of general type, Invent. Math. 166 (2006), 1-25.

[9] K. Ohno, Some inequalities for minimal fibrations of surfaces of general type over curves. J. Math. Soc. Japan 44(4) (1992), 643-666.

[10] M. Reid, Young person's guide to canonical singularities, Proc. Sympos. Pure Math. 46 (1987), $345-414$.

[11] S. Takayama, Pluricanonical systems on algebraic varieties of general type, Invent. Math. 165 (2006), 551-587.

[12] H. Tsuji, Pluricanonical systems of projective varieties of general type, I, Osaka J. Math. 43 (2006), 967-995.

[13] E. Viehweg, Weak positivity and the additivity of the Kodaira dimension for certain fibre spaces, in 'Adv. Studies in Math.' (Proc. Algebraic Varieties and Analytic Varieties, Tokyo, 1981), 1, Kinokunya-North-Holland Publ. 1983, 329-353. 
National Center for Theoretical Sciences, Taipei Office and Department of Mathematics, National Taiwan University, Taipei 106, Taiwan

E-mail address: jkchen@math.ntu.edu.tw

Institute of Mathematics \& LMnS, Fudan University, Shanghai 200433, People's Republic of China

E-mail address: mchen@fudan.edu.cn

Mathématiques BÂtiment 425, Université Paris-Sud, F-91405 Orsay, France

E-mail address: zhi.jiang@math.u-psud.fr 
